\title{
Rapid Extract Dereplication Using HPLC-SPE-NMR: Analysis of Isoflavonoids from Smirnowia iranica
}

Maja Lambert, Dan Stærk, Steen Honoré Hansen, Majid Sairafianpour, and Jerzy W. Jaroszewski

\section{Supporting Information}

List of NOESY and HMBC correlations observed for compounds 8, 10, 12, 13 and 15 (chemical shift values are reported in the article). In addition, HSQC spectra of all compounds were recorded and showed the expected 1-bond correlations. All spectra were recorded at $600 \mathrm{MHz}$ in $\mathrm{CDCl}_{3}$ with TMS as internal standard.

\section{Compound 8}

\begin{tabular}{|c|c|c|}
\hline Hydrogen & NOESY & HMBC \\
\hline $\mathrm{H}-2$ & $\mathrm{H}-3$ & C-3; C-4; C-9; C-1' \\
\hline $\mathrm{H}-3$ & $\mathrm{H}-2$ & C-2; C-4; C-1'; C-2'; C-6' \\
\hline H-5 & $\mathrm{H}-6$ & $\mathrm{C}-4 ; \mathrm{C}-7 ; \mathrm{C}-8 ; \mathrm{C}-10$ \\
\hline H-6 & $\mathrm{H}-5$ & C-7; C-8; C-10 \\
\hline H-5' & $\mathrm{H}-6^{\prime} ; \mathrm{OCH}_{3}-4^{\prime}$ & C-1'; C-2'; C-3'; C-4' \\
\hline H-6' & H-5' & C-3; C-2'; C-3'; C-4' \\
\hline $\mathrm{H}-1 "$ & $\mathrm{H}-2 " ; \mathrm{CH}_{3}-3^{\prime \prime}$ & C-7; C-8; C-9; C-2"; C-3" \\
\hline $\mathrm{H}-2^{\prime \prime}$ & $\mathrm{H}-1 " ; \mathrm{CH}_{3}-3 "$ & $\mathrm{C}-1 " ; \mathrm{CH}_{3}-3 "$ \\
\hline $\mathrm{CH}_{3}-3 "$ & H-1"; H-2" & C-8; C-2"; C-3" \\
\hline $\mathrm{OCH}_{3}-4^{\prime}$ & H-5' & C- $4^{\prime}$ \\
\hline
\end{tabular}

\section{Compound 10}

\begin{tabular}{|c|c|c|}
\hline Hydrogen & NOESY & HMBC \\
\hline $\mathrm{H}-2 \mathrm{~A}$ & $\mathrm{H}-2 \mathrm{~B} ; \mathrm{H}-3$ & $C-3 \cdot C-9$ \\
\hline $\mathrm{H}-2 \mathrm{~B}$ & $\mathrm{H} 2 \mathrm{~A} ; \mathrm{H}-3 ; \mathrm{H}-4 \mathrm{~B}$ & 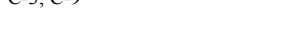 \\
\hline $\mathrm{H}-3$ & $\mathrm{H}-2 \mathrm{~A} ; \mathrm{H}-2 \mathrm{~B} ; \mathrm{H}-4 \mathrm{~A} ; \mathrm{H}-4 \mathrm{~B} ; \mathrm{H}-6^{\prime} ; \mathrm{OCH}_{3}-2^{\prime}$ & C-2; C-4; C-1'; C-2'; C-6' \\
\hline $\mathrm{H}-4 \mathrm{~A}$ & $\mathrm{H}-3 ; \mathrm{H}-4 \mathrm{~B} ; \mathrm{H}-5 ; \mathrm{H}-6^{\prime}$ & $C_{-} 2 \cdot C_{-3} \cdot C_{-} 5 \cdot C_{-} \cdot C_{-10}$ \\
\hline H-4B & H-2B; H-3; H-4A; H-5; H-6' & 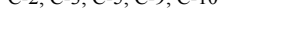 \\
\hline H-5 & $\mathrm{H}-4 \mathrm{~A} ; \mathrm{H}-6$ & C-4; C-7; C-9 \\
\hline H-6 & H-5 & C-7; C-8; C-10 \\
\hline H-5' & $\mathrm{H}-6^{\prime} ; \mathrm{OCH}_{3}-4^{\prime}$ & C-1'; C-3'; C-4' \\
\hline H-6' & H-2B; H-4B; H-5' & C-3; C-4; C-2'; C-3'; C-4' \\
\hline H-1" & $\mathrm{H}-2 " ; \mathrm{CH}_{3}-3 "$ & $\mathrm{C}-8 ; \mathrm{C}-2 " ; \mathrm{CH}_{3}-3 "$ \\
\hline H-2" & $\mathrm{H}-1 " ; \mathrm{CH}_{3}-3^{\prime \prime}$ & C-7; C-8; C-9; C-1"; C-3" \\
\hline $\mathrm{CH}_{3}-3 "$ & H-1"; H-2" & C-1"; C-3" \\
\hline $\mathrm{OCH}_{3}-2^{\prime}$ & $\mathrm{H}-3$ & C-2' \\
\hline $\mathrm{OCH}_{3}-4^{\prime}$ & H-5' & C-4' \\
\hline
\end{tabular}

\section{Compound 12}

\begin{tabular}{|c|c|c|}
\hline Hydrogen & NOESY & HMBC \\
\hline $\mathrm{H}-2$ & H-2'; H-1"; $\mathrm{CH}_{3}-3^{\prime \prime}$ & C-3; C-4; C-9; C-1' \\
\hline H-5 & $\mathrm{H}-6$ & C-4; C-7; C-9 \\
\hline H-6 & $\mathrm{H}-5$ & C-7; C-8; C-10 \\
\hline H-2' & $\mathrm{H}-2$ & C-3; C-3'; C-4'; C-6' \\
\hline H-5' & $\mathrm{H}-6^{\prime} ; \mathrm{OCH}_{3}-4^{\prime}$ & C-1'; C-3'; C-4' \\
\hline H-6' & $\mathrm{H}-5^{\prime}$ & C-3; C-2'; C-3'; C-4' \\
\hline $\mathrm{H}-\mathrm{l}^{\prime}$ & $\mathrm{H}-2^{\prime \prime} ; \mathrm{CH}_{3}-3 "$ & C-7; C-8; C-9; C-2"; C-3" \\
\hline H-2" & $\mathrm{H}-1 " ; \mathrm{CH}_{3}-3 "$ & $\mathrm{C}-8 ; \mathrm{C}-1 " ; \mathrm{CH}_{3}-3 "$ \\
\hline $\mathrm{CH}_{3}-3 "$ & $\mathrm{H}-2 ; \mathrm{H} 1 " ; \mathrm{H} 2 "$ & C-2"; C3" \\
\hline $\mathrm{OCH}_{3}-4^{\prime}$ & $\mathrm{H}-5^{\prime}$ & C-4' \\
\hline
\end{tabular}




\section{Compound 13}

\begin{tabular}{|c|c|c|}
\hline Hydrogen & NOESY & HMBC \\
\hline $\mathrm{H}-2 \mathrm{~A}$ & $\mathrm{H}-2 \mathrm{~B} ; \mathrm{H}-3 ; \mathrm{OCH}_{3}-2^{\prime}$ & \multirow{2}{*}{ C-3; C-4; C-9 } \\
\hline $\mathrm{H}-2 \mathrm{~B}$ & H2A; H-3; H-4B; H-6' & \\
\hline $\mathrm{H}-3$ & $\mathrm{H}-2 \mathrm{~A} ; \mathrm{H}-2 \mathrm{~B} ; \mathrm{H}-4 \mathrm{~A} ; \mathrm{H}-4 \mathrm{~B} ; \mathrm{OCH}_{3}-2^{\prime}$ & $\mathrm{C}-2 ; \mathrm{C}-1^{\prime} ; \mathrm{C}-2^{\prime}$ \\
\hline $\mathrm{H}-4 \mathrm{~A}$ & H-4B; H-3; H-5; H-6' & \multirow{2}{*}{ C-2; C-3; C-9; C-10, C-1' } \\
\hline H-4B & Н-2B; H-4A; H-3; H-5; H-6' & \\
\hline $\mathrm{H}-5$ & H4A; H4B; H-6 & C-4; C-7; C-9 \\
\hline H-6 & $\mathrm{H}-5$ & C-8; C-10 \\
\hline H-5' & $\mathrm{H} 6$ '; $\mathrm{OCH}_{3}-4$ ' & $\mathrm{C}-1^{\prime} ; \mathrm{C}-3^{\prime} ; \mathrm{C}-6^{\prime}$ \\
\hline $\mathrm{H}-6^{\prime}$ & H2B; H-3; H-4A; H-4B; H5' & C-3; C-2'; C-4' \\
\hline $\mathrm{H}-1^{\prime}$ & $\mathrm{H}-3 ; \mathrm{H}-4 " \mathrm{Z}$ & C-7; C-8; C-9: C-2"; C-3" \\
\hline $\mathrm{CH}_{3}-3 "$ & H-1"; H-4"E & C-2"; C-3"; C-4" \\
\hline H-4"E & $\mathrm{CH}_{3}-3 " ; \mathrm{H}-4 " \mathrm{Z}$ & \multirow{2}{*}{$\mathrm{C}-2^{\prime \prime} ; \mathrm{C}-3 " ; \mathrm{CH}_{3}-3 "$} \\
\hline $\mathrm{H}-4 " \mathrm{Z}$ & H-4"E & \\
\hline $\mathrm{OCH}_{3}-2^{\prime}$ & $\mathrm{H}-2 \mathrm{~A} ; \mathrm{H}-3$ & $\mathrm{C}-2^{\prime}$ \\
\hline $\mathrm{OCH}_{3}-4$ & $\mathrm{H}-5^{\prime}$ & C-4' \\
\hline
\end{tabular}

\section{Compound 15}

\begin{tabular}{|c|c|c|}
\hline Hydrogen & NOESY & HMBC \\
\hline $\mathrm{H}-2 \mathrm{~A}$ & $\mathrm{H}-2 \mathrm{~B} ; \mathrm{H}-3 ; \mathrm{CHO}-8$ & \multirow{2}{*}{ C-3; C-4; C-9; C-1' } \\
\hline $\mathrm{H}-2 \mathrm{~B}$ & H2A; H-3; H-4B; H-6'; CHO-8 & \\
\hline $\mathrm{H}-3$ & $\mathrm{H}-2 \mathrm{~A} ; \mathrm{H}-2 \mathrm{~B} ; \mathrm{H}-4 \mathrm{~A} ; \mathrm{H}-4 \mathrm{~B} ; \mathrm{H}-6^{\prime} ; \mathrm{OCH}_{3}-2^{\prime}$ & C-2; C-4; C-1'; C-2'; C-6' \\
\hline $\mathrm{H}-4 \mathrm{~A}$ & H-4B; H-3; H-5; H-6' & \multirow{2}{*}{ C-2; C-3; C-5; C-9; C-10, C-1' } \\
\hline $\mathrm{H}-4 \mathrm{~B}$ & H-2B; H-4A; H-3; H-5; H-6' & \\
\hline $\mathrm{H}-5$ & $\mathrm{H} 4 \mathrm{~A} ; \mathrm{H} 4 \mathrm{~B} ; \mathrm{H}-6 ; \mathrm{OCH}_{3}-4^{\prime}$ & C-4; C-7; C-8; C-9 \\
\hline H-6 & $\mathrm{H}-5 ; \mathrm{OH}-7$ & C-7; C-8; C-10 \\
\hline H-5' & $\mathrm{H} 2 \mathrm{~A} ; \mathrm{H} 2 \mathrm{~B} ; \mathrm{H6}^{\prime} ; \mathrm{OCH}_{3}-4^{\prime}$ & C-1'; C-2'; C-3'; C-4' \\
\hline H-6' & $\mathrm{H}-2 \mathrm{~A} ; \mathrm{H}-2 \mathrm{~B} ; \mathrm{H}-3 ; \mathrm{H}-4 \mathrm{~A} ; \mathrm{H}-4 \mathrm{~B} ; \mathrm{H} 5^{\prime} ; \mathrm{OCH}_{3}-2^{\prime}$ & C-3; C-2'; C-3'; C-4' \\
\hline $\mathrm{OCH}_{3}-2^{\prime}$ & H-3; H-6' & $\mathrm{C}-2^{\prime}$ \\
\hline $\mathrm{OCH}_{3}-4^{\prime}$ & H-5; H-5' & C-4' \\
\hline CHO-8 & $\mathrm{H}-2 \mathrm{~A} ; \mathrm{H}-2 \mathrm{~B}$ & C-6; C-7; C-8 \\
\hline $\mathrm{OH}-7$ & H-6 & C-5; C-6; C-7; C-8 \\
\hline
\end{tabular}

${ }^{1}$ H NMR Data for Compounds 3, 6, 11, 14 and 17 in Acetonitrile- $\boldsymbol{d}_{3}$. The spectra were recorded with previously isolated compounds ${ }^{13}$ as a reference for HPLC-SPE-NMR analysis. Compound 3 (600 MHz, $\left.\mathrm{CD}_{3} \mathrm{CN}\right): \delta 1.87\left(3 \mathrm{H}, \mathrm{dd}, J=1.7\right.$ and $\left.6.5 \mathrm{~Hz}, \mathrm{CH}_{3}\right), 3.68\left(3 \mathrm{H}, \mathrm{s}, \mathrm{OCH}_{3}-6\right), 3.77\left(3 \mathrm{H}, \mathrm{s}, \mathrm{OCH}_{3}-3\right), 3.80(3 \mathrm{H}$, s, $\left.\mathrm{OCH}_{3}-2\right), 6.25(1 \mathrm{H}, \mathrm{dq}, J=15.8$ and $6.5 \mathrm{~Hz}, \mathrm{H}-2$ '), $6.56(1 \mathrm{H}, \mathrm{s}, \mathrm{H}-4), 6.59(1 \mathrm{H}, \mathrm{dq}, J=15.8$ and $1.7 \mathrm{~Hz}$, H-1'); compound $6\left(600 \mathrm{MHz}, \mathrm{CD}_{3} \mathrm{CN}\right): \delta 1.89\left(3 \mathrm{H}, \mathrm{dd}, J=1.7\right.$ and $\left.6.9 \mathrm{~Hz}, \mathrm{CH}_{3}\right), 3.91,3.92$ and 3.93 (each $\left.3 \mathrm{H}, \mathrm{s}, \mathrm{OCH}_{3}\right), 6.34\left(1 \mathrm{H}, \mathrm{dq}, J=16.0\right.$ and $\left.1.7 \mathrm{~Hz}, \mathrm{H}-1^{\prime}\right), 6.84\left(1 \mathrm{H}, \mathrm{dq}, J=16.0\right.$ and $\left.6.9 \mathrm{~Hz}, \mathrm{H}-2^{\prime}\right)$; compound $11\left(600 \mathrm{MHz}, \mathrm{CD}_{3} \mathrm{CN}\right): \delta 1.36$ and 1.37 (each $\left.3 \mathrm{H}, \mathrm{s}, \mathrm{CH}_{3}\right), 2.82(1 \mathrm{H}$, ddd, $J=15.6,5.0$ and 2.0

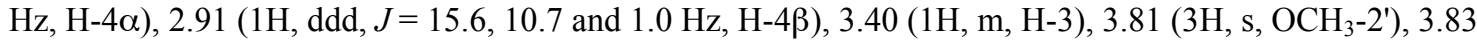
$\left(3 \mathrm{H}, \mathrm{s}, \mathrm{OCH}_{3}-4\right.$ '), $3.95(1 \mathrm{H}, \mathrm{t}, J=10.4 \mathrm{~Hz}, \mathrm{H}-2 \beta), 4.27(1 \mathrm{H}, \mathrm{ddd}, J=10.4,3.5$ and $2.0 \mathrm{~Hz}, \mathrm{H}-2 \alpha), 5.63(1 \mathrm{H}$, d, $J=9.9 \mathrm{~Hz}, \mathrm{H}-2 "), 6.29$ (1H, d, $J=8.3 \mathrm{~Hz}, \mathrm{H}-6), 6.59$ (1H, d, $J=9.9 \mathrm{~Hz}, \mathrm{H}-1 "), 6.63$ (1H, d, $J=8.6 \mathrm{~Hz}$, H-5'), $6.71\left(1 \mathrm{H}, \mathrm{d}, J=8.6 \mathrm{~Hz}, \mathrm{H}-6^{\prime}\right), 6.84(1 \mathrm{H}, \mathrm{d}, J=8.3 \mathrm{~Hz}, \mathrm{H}-5)$; compound $14\left(600 \mathrm{MHz}, \mathrm{CD}_{3} \mathrm{CN}\right): \delta$ $2.97(1 \mathrm{H}, \mathrm{ddd}, J=15.6,5.0$ and $2.0 \mathrm{~Hz} \mathrm{H}-4 \alpha), 3.07$ (1H, ddd, $J=15.6,10.7$ and $1.0 \mathrm{~Hz}, \mathrm{H}-4 \beta), 3.55(1 \mathrm{H}$, m, H-3), $3.83\left(3 \mathrm{H}, \mathrm{s}, \mathrm{OCH}_{3}-2^{\prime}\right), 3.84\left(3 \mathrm{H}, \mathrm{s}, \mathrm{OCH}_{3}-4{ }^{\prime}\right), 4.12(1 \mathrm{H}, \mathrm{t}, J=10.6 \mathrm{~Hz}, \mathrm{H}-2 \beta), 4.36(1 \mathrm{H}, \mathrm{ddd}, J=$ 
10.6, 3.7 and $2.0 \mathrm{~Hz}, \mathrm{H}-2 \alpha), 6.66\left(1 \mathrm{H}, \mathrm{d}, J=8.6 \mathrm{~Hz}, \mathrm{H}-5^{\prime}\right), 6.72\left(1 \mathrm{H}, \mathrm{d}, J=8.6 \mathrm{~Hz}, \mathrm{H}-6^{\prime}\right), 6.82(1 \mathrm{H}, \mathrm{dd}, J=$ 2.2 and $1.0 \mathrm{~Hz}, \mathrm{H}-1 "), 7.06(1 \mathrm{H}, \mathrm{dd}, J=8.6$ and $1.0 \mathrm{~Hz}, \mathrm{H}-6), 7.05(1 \mathrm{H}, \mathrm{d}, J=8.6, \mathrm{H}-5), 7.63(1 \mathrm{H}, \mathrm{d}, J=$ $\left.2.2 \mathrm{~Hz}, \mathrm{H}-2^{\prime \prime}\right)$; compound 17 (600 MHz, $\mathrm{CD}_{3} \mathrm{CN}$ ): $\delta 1.66$ and 1.74 (each $3 \mathrm{H}$, br s, $\left.\mathrm{CH}_{3}-3 "\right)$, 2.82 (1H, ddd, $J=15.6,5.0$ and $2.0 \mathrm{~Hz} \mathrm{H}-4 \alpha), 2.90(1 \mathrm{H}, \mathrm{ddd}, J=15.6,10.7$ and $1.0 \mathrm{~Hz}, \mathrm{H}-4 \beta), 3.25(2 \mathrm{H}, \mathrm{d}, J=7.0 \mathrm{~Hz}$, H-1"), 3.40 (1H, m, H-3), $3.81\left(3 \mathrm{H}, \mathrm{s}, \mathrm{OCH}_{3}-2^{\prime}\right), 3.83\left(3 \mathrm{H}, \mathrm{s}, \mathrm{OCH}_{3}-4^{\prime}\right), 3.95$ (1H, t, $\left.J=10.6 \mathrm{~Hz}, \mathrm{H}-2 \beta\right)$, 4.24 (1H, ddd, $J=10.6,3.7$ and $2.0 \mathrm{~Hz}, \mathrm{H}-2 \alpha), 5.16$ (1H, tsp, $J=7.0$ and $1.0 \mathrm{~Hz}, \mathrm{H}-2 "), 6.35$ (1H, d, $J=$ $8.3 \mathrm{~Hz}, \mathrm{H}-6), 6.63$ (1H, d, J=8.6 Hz, H-5'), 6.70 (1H, d, J=8.6 Hz, H-6'), 6.75 (1H, d, J= 8.3 Hz, H-5).

The stereochemical assignments of the C-ring hydrogens in $\mathbf{1 1}, \mathbf{1 4}$, and $\mathbf{1 7}$ follow from the previous work. ${ }^{13}$

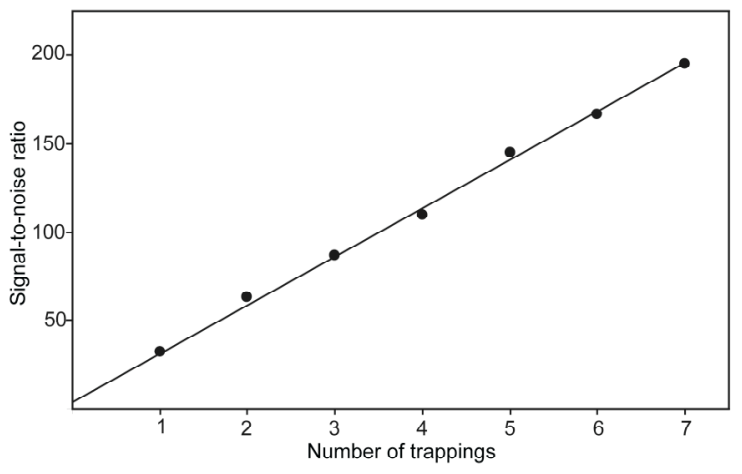

Figure S1. Linear increase of signal-to-noise ratios in ${ }^{1} \mathrm{H}$ NMR spectra of $\mathbf{1 1}$ after repeated trappings on the same SPE cartridge; S/N was calculated for spectral regions shown in Figure 4. 